\title{
Numerical modeling of the labyrinth seal taking into account vibrations of the gas transmittal unit rotor in aeroelastic formulation
}

\author{
L.N. Butymova ${ }^{1}$, V.Ya. Modorskii ${ }^{1}$ \\ ${ }^{I}$ Perm National Research Polytechnic University, pr. Komsomolsky 29, 614099,Perm, Russia
}

\begin{abstract}
The article deals with the issues related to the mutual influence of vibrations and gas dynamic processes in the labyrinth seals (LS) of gas transmittal unit compressors. The mutual influence of vibrational gas dynamic processes in LS and vibrations of the rotor is studied. Within the framework of the unified algorithm, a solution is obtained for an unsteady aeroelastic one-dimensional gas flow problem in a deformable LS. A new factor (the rotor diameter in the LS region), which affects the pulsation magnitude of the gas dynamic force in the LS, is revealed. Changing the diameter of the rotor, you can reduce vibration. In this case, it is possible to reduce the designated clearances in the LS and to reduce the leakage.
\end{abstract}

Keywords: aeroelasticity; rotor vibration; labyrinth seal; unified algorithm; stress; pressure; deformation; displacement

\section{Introduction}

To ensure a contactless connection between a rotating rotor and a stationary body in aircraft engines [16], high-pressure pumps [13, 14], etc., labyrinth seals (LS) are used. In seals of labyrinth type, the working medium is sealed by throttling it when moving through successively located constrictions and extensions.

The main task of the LS is to ensure tightness of the rotor, therefore expansions and constrictions of the flow in LS are usually considered in the direction parallel to the rotor axis. However, in order to ensure aerovibration resistance, it is necessary to take into account the processes of motion of the working medium that take place in the peripheral direction of the LS under the rotor vibrations. It should be noted that sequencing of the constrictions and expansions affects the oscillation amplitude in the gas-dynamic cavity between the LS and the rotor, and also increases the flow non-uniformity. Consequently, refusal to take these elements into account in aeroelastic calculation [15, 21-22] can give an additional margin from the point of view of reducing oscillations in LS, and, which is important for solving the related problems of continuum mechanics [19], reduce complexity and time of calculations.

Based on the said above, the LS calculation is replaced by calculating the gap seal, equivalent (with margin) to the labyrinth seal, if we consider the processes taking place in the LS circumferential direction.

As it is known,LS works at high temperatures and high rotation speeds. Under critical operating conditions the LS is effected by significant loads from the gas-dynamic flow as well as the LS influences the gas-dynamic flow. The impact of this process is ambiguous and requires more detailed research. Publications related to vibrational processes in LS and vibration of rotors, consider the influence of precession [11], geometric characteristics of LS [12] and in the most cases do not take into account the influence of gas-dynamic forces.

The gas dynamic processes that arise in LS at the rotor vibrations caused, for example, by technological imbalances, may lag behind the rotor oscillations. It is necessary to analyze the possibility of amplifying or weakening the rotor vibrations and dependence of these processes on the LS characteristics [9-10].

The classical formulation of the vibration problem takes into account the influence of structural [20], physical-mechanical and technological parameters on vibration, but does not consider the influence of gas dynamic loads.

When considering the problem of the vibration effect on gas-dynamic processes in the labyrinth seals of a centrifugal compressor model gas transmittal unit in a unidirectional dynamic related formulation it is possible to take into account the gasdynamic factors $[13,17]$. In addition, it becomes possible to calculate the oscillation parameters of gas-dynamic forces acting on the rotor [4-8].

\section{Object of study}

A physical model describing the LS operation in an aeroelastic formulation is developed. As a model, the LS scan with the width corresponding to the LS width is considered. In a unidirectional FSI-statement, the rotor motion is replaced by the movement of pistons located diametrically opposite, and moving with the specified amplitude and frequency (Fig.1). The scan length is equal to the circumference of the gas-dynamic cavity, which is aligned along the middle line between the rotating rotor and the stationary LS.

The model is quasi-two-dimensional, dynamic.

Thus, the rotor oscillations in the LS gap are modeled by a nonlinear dynamic quasi-two-dimensional gas dynamic scan model of the LS gap with movable boundaries. Extrusion of the gas as the gap is reduced and filling free volume with the gas as the gap is increased during the rotor oscillation in the LS is modeled by two pistons moving in harmonic order. Their oscillation frequency is the same and corresponds to the rotor oscillation frequency, calculated from the wave path equal to the length of the circumference arc around the rotor. The piston oscillation amplitudes are equal (formula 1). While calculating, the 
oscillations of displacements, velocities, pressures and gas-dynamic force acting on the rotor in the LS area are recorded at the control points. The displacement of these oscillations $\left(\varphi_{\mathrm{U}}\right)$ may take place with respect to two parameters: the gas-dynamic forces acting on the rotor in the LS area and the rotor displacements. With different phases $\left(\varphi_{U}\right)$ convergent, divergent and steady oscillatory processes can be observed.

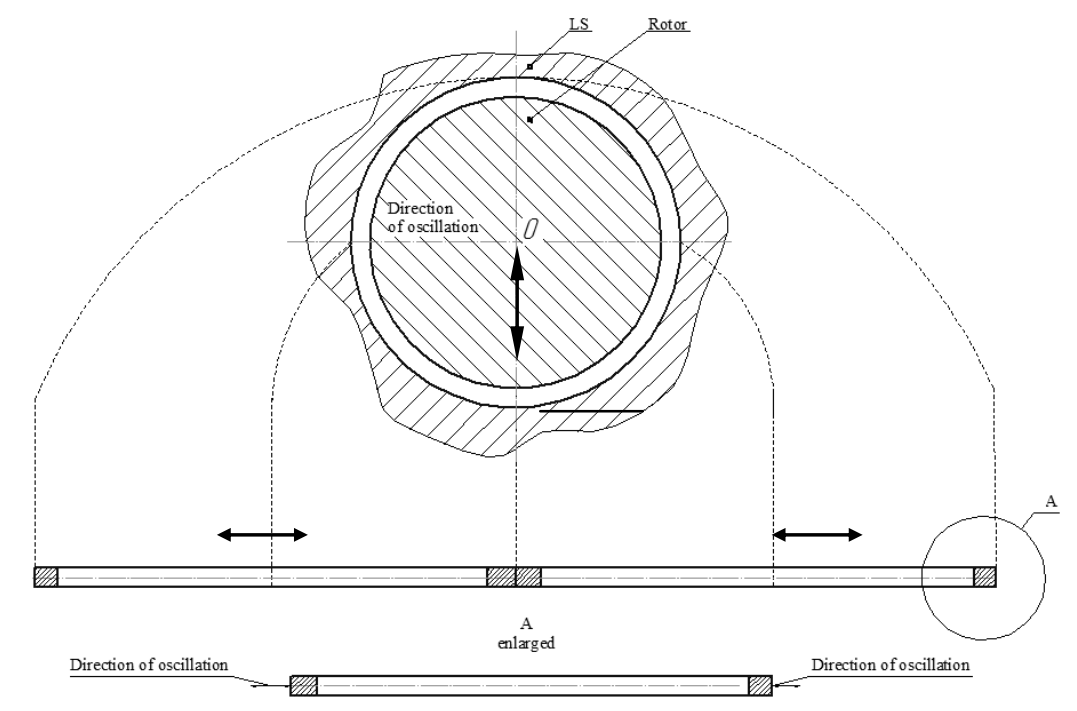

Fig. 1. Formation of the LS calculation scheme (unidirectional FSI-statement).

\section{Mathematical model}

The mathematical description of the gas-elastic process in this formulation includes the following relationships:

$$
\begin{aligned}
& \frac{\partial \rho_{\Gamma}}{\partial t}+\frac{\partial \rho_{\Gamma} V_{x}}{\partial x}=0 \\
& \frac{\partial \rho_{\Gamma} V_{x \Gamma}}{\partial t}+\frac{\partial\left(\rho_{\Gamma} V_{x \Gamma} V_{x \Gamma}\right)}{\partial x}+\frac{\partial P}{\partial x}=0 \\
& \frac{\partial \rho_{\Gamma} E}{\partial t}+\frac{\partial\left(\rho_{\Gamma} E V_{x \Gamma}\right) \rho_{\Gamma} E}{\partial x}+\frac{\partial\left(P V_{x}\right)}{\partial x}=0 \\
& P=\rho_{\Gamma}(k-1)\left(E-V_{x \Gamma}^{2} / 2\right) \\
& \frac{\partial \rho_{\mathrm{K}}}{\partial t}+\frac{\partial\left(\rho_{\mathrm{K}} V_{x \mathrm{~K}}\right)}{\partial x}=0 \\
& \frac{\partial\left(\rho_{\mathrm{K}} V_{x \mathrm{~K}}\right)}{\partial t}-\frac{\partial \sigma_{x x}}{\partial x}=0 \\
& U_{x}=U_{x 0}+\int_{0}^{t} V_{x \mathrm{~K}}(t) d t \\
& \varepsilon_{x x}=\frac{\partial U_{x}}{\partial x} \\
& \sigma_{x x}=E \varepsilon_{x x} \\
& - \text { initial conditions } \\
& \text { at } t=0(\mathrm{Gas}): \\
& P=P \\
& 0, \rho_{\Gamma}=\rho_{0 \Gamma}, E=P_{0} / \rho_{0 \Gamma}(k-1) \\
& \text { at } t=0(\mathrm{SSS}): \\
& \sigma_{x x}=0, \varepsilon_{x x}=0, U_{x 0}=0, V_{x}=0 \\
& x_{x y}
\end{aligned}
$$

Boundary conditions (SSS)
a) «rigid wall»

\section{$(«$ Sticking»)}

$V_{x \kappa}=0$,

$$
V_{x \kappa}=0
$$


a) gas-structure

$$
\sigma_{x x}=-P_{\Gamma \mathrm{p}} \quad V_{x \kappa}=V_{x \Gamma}
$$

The boundary condition for the piston motion:

$$
\mathrm{V}_{\text {left.piston }}=-\mathrm{V}_{0} \sin (\omega t) ; \mathrm{V}_{\text {right.piston }}=\mathrm{V}_{0} \sin (\omega t)
$$

where $V_{0}$ - amplitude of the piston oscillations, $\omega$ - the piston oscillation frequency.

\section{Method of solution}

For solving gas dynamic tacks was used method of large particles. Using the same method for solving gas dynamic and stress-strain state tasks provide unity mesh for gas dynamic region and stress-strain state tasks. For this used unified system of differential equations to ensurecoupled solving for elastic tasks and gas dynamic tasks. Thus we used method of large particles for calculations.

The main idea of method's large particles consisted in splitting into physical processes of the initial non-stationary system of Euler equations which written in the form's conservation laws. The space is modeled by particle system which coincides with cell's Euler grid in the moment. If stationary solving is, we get it in process stabilized solving. So all process solving composed multiple repetitions of time steps.

Each computational cycle is divided into seven stages. The first three stages are designed to solve the gas dynamic tasks. The next four stages are designed to evaluate the parameters dynamic stress-strain state of the structure.

\section{Description of results}

\subsection{Analysis of the influence of geometric characteristics}

In a unidirectional aeroelastic formulation, the solution using equations (1-4) and initial and boundary conditions, yielded the following results.

When investigating the dependence of pressure fluctuations in the LS gas-dynamic cavity on the LS geometric characteristics the rotor diameters in the LS area variedand were equal to $65,130,195$ or $260 \mathrm{~mm}$. The working body of the gas-dynamic cavity is air, adiabatic exponent equals 1.4 , density $\rho=1,29 \mathrm{~kg} / \mathrm{m}^{3}$.

With an increase in the shaft diameter from $65 \mathrm{~mm}$ to $260 \mathrm{~mm}$, the pressure oscillations in the gas-dynamic cavity are noted, which have a time periodic character (Fig. 2). The pressure amplitude is $101368.8 \mathrm{~Pa}$ with $\mathrm{D}=65 \mathrm{~mm}$, with $\mathrm{D}=130 \mathrm{~mm}$ the pressure amplitude is $148792.2 \mathrm{~Pa}$, and with $\mathrm{D}=195 \mathrm{~mm}$ the pressure amplitude is $107577.7 \mathrm{~Pa}$, with $\mathrm{D}=260 \mathrm{~mm}$ the pressure amplitude is $101732.1 \mathrm{~Pa}$.

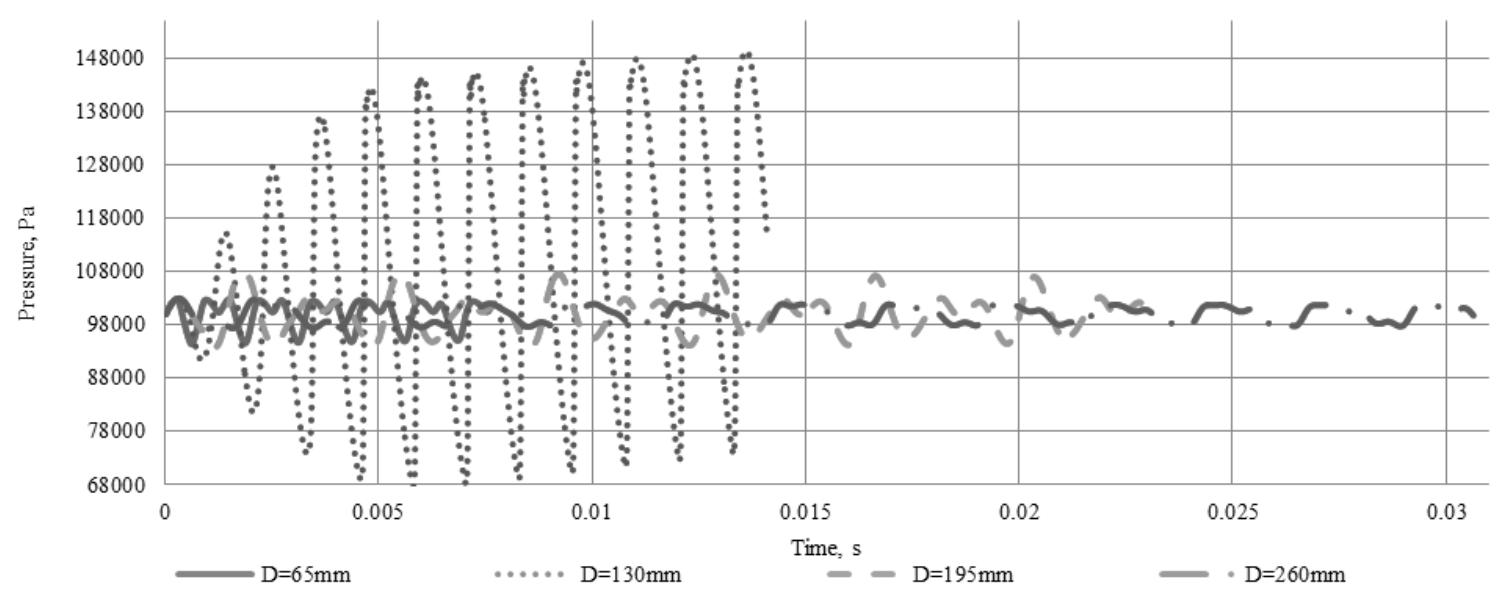

Fig. 2. Dependence of the gas dynamic pressure of the gap near LS on time at different geometric characteristics of LS.

Table 1. Pressure amplitude for LS different geometric characteristics.

\begin{tabular}{ccccc}
\hline Computational experiment & 1 & 2 & 3 & 4 \\
\hline Rotor diameter D, mm & 65 & 130 & 195 & 260 \\
\hline $\begin{array}{c}\text { Maximum pressure amplitude } \\
\text { U }_{\mathrm{P}, \mathrm{MPa}}\end{array}$ & 0.1 & 0.15 & 0.11 & 0.102 \\
\hline
\end{tabular}

When studying the dependence of the temperature oscillations in the gas-dynamic cavity on the geometric characteristics of the LU, the diameters of the rotor in the zone of the LU varied and assumed values of 65, 130, 195 or $260 \mathrm{~mm}$, the working fluid of the gas-dynamic cavity is air, the adiabatic index is $1.4, \rho=1.29 \mathrm{~kg} / \mathrm{m}^{3}$.

With an increase in shaft diameter from $65 \mathrm{~mm}$ to $260 \mathrm{~mm}$, oscillations in the temperature of the gas-dynamic cavity are noted, which have a periodic character of the change in time (Fig. 3). The temperature amplitude is $272.4936 \mathrm{~K}$. At D $=65 \mathrm{~mm}$, 


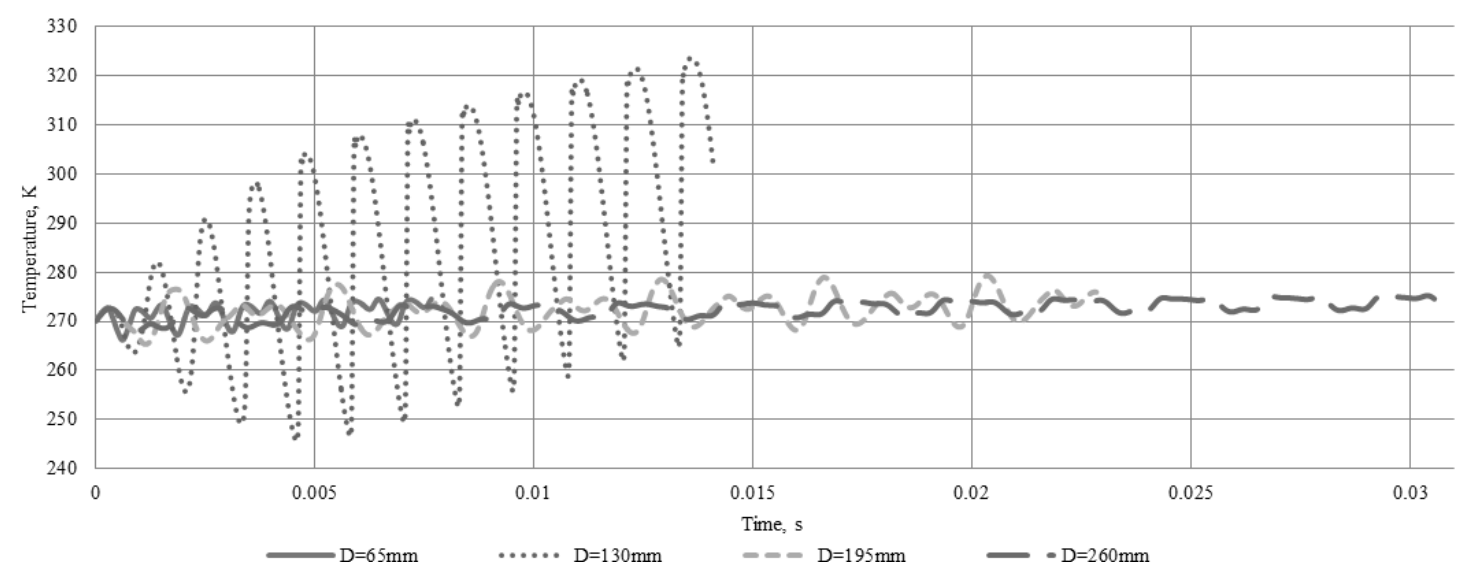

Fig.3. Dependence of the gas-dynamic gap temperature near LS on the time for LS different geometric characteristics.

Thus, when designing the LS it is necessary to take into account the geometric dimensions of the rotor and the gap between the rotor and the LS in order to reduce possible vibrations.

Table 2. Temperature amplitude for LS different geometric characteristics.

\begin{tabular}{|c|c|c|c|c|}
\hline Computational experiment & 1 & 2 & 3 & 4 \\
\hline Rotor diameter $\mathrm{D}, \mathrm{mm}$ & 65 & 130 & 195 & 260 \\
\hline Temperature amplitude $\mathrm{U}_{\mathrm{T}} \mathrm{K}$ & 272.4936 & 323.6043 & 279.0668 & 274.4728 \\
\hline
\end{tabular}

\subsection{Analysis of the influence of kinematic parameters}

The dependence of pressure oscillations in the LS gas dynamic cavity on the kinematic parameters of propagation speed of gas oscillations in the circumferential direction varied and equaled $3.5,7.0$ or $10.5 \mathrm{~m} / \mathrm{s}$, the working fluid of the gas-dynamic cavity is air, the adiabatic index is $1.4, \rho=1,29 \mathrm{~kg} / \mathrm{m}^{3}$.

With speed increase from $3.5 \mathrm{~m} / \mathrm{s}$ to $10.5 \mathrm{~m} / \mathrm{s}$, the pressure oscillations of the gas-dynamic cavity are noted, which have a periodic character (Fig. 4). The pressure amplitude is $100918.3 \mathrm{~Pa}$ at $\mathrm{V}=3.5 \mathrm{~m} / \mathrm{s}$. At $\mathrm{V}=7.0 \mathrm{~m} / \mathrm{s}$, the pressure amplitude is $103,000 \mathrm{~Pa}$, and at $\mathrm{V}=10.5 \mathrm{~m} / \mathrm{s}$ the pressure amplitude is $104419 \mathrm{~Pa}$.

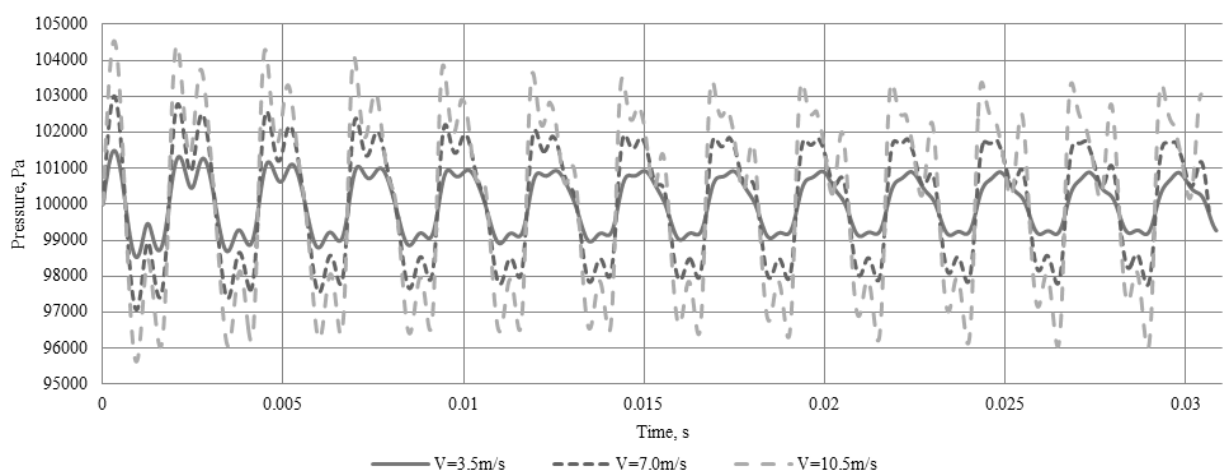

Fig.4. Pressure dependence in the gas-dynamic gap near the LS on time for different elasticity moduli of LS material.

Table 3. Pressure amplitude at LS different kinematic parameters.

\begin{tabular}{|c|c|c|c|}
\hline Computational experiment & 1 & 2 & 3 \\
\hline $\begin{array}{l}\text { Propagation speed of gas } \\
\text { oscillations in the LS } \\
\text { circumferential direction, } \mathrm{m} / \mathrm{s}\end{array}$ & 3.5 & 7.0 & 10.5 \\
\hline $\begin{array}{c}\text { Maximum pressure amplitude } \\
\mathrm{U}_{\mathrm{P}, \mathrm{MPa}}\end{array}$ & 0.101 & 0.103 & 0.104 \\
\hline
\end{tabular}

When studying the temperature dependence of the gas dynamic cavity in the LS on kinematic parameters, the propagation speed of the gas oscillations in the circumferential direction varied and assumed values of 3.5, 7.0 or $10.5 \mathrm{~m} / \mathrm{s}$, the working fluid of the gas-dynamic cavity is air, adiabatic index is 1.4 , air density $\rho=1,29 \mathrm{~kg} / \mathrm{m}^{3}$. 
With speed increase from $3.5 \mathrm{~m} / \mathrm{s}$ to $10.5 \mathrm{~m} / \mathrm{s}$ oscillations in the temperature of the gas-dynamic cavity are observed, which have a periodic character (Fig. 5). The temperature amplitude is $271.9472 \mathrm{~K}$ at $\mathrm{V}=3.5 \mathrm{~m} / \mathrm{s}$. At $\mathrm{V}=7.0 \mathrm{~m} / \mathrm{s}$ the temperature amplitude is $275.1519 \mathrm{~K}$, and at $\mathrm{V}=10.5 \mathrm{~m} / \mathrm{s}$ the temperature amplitude is $279.8211 \mathrm{~K}$.

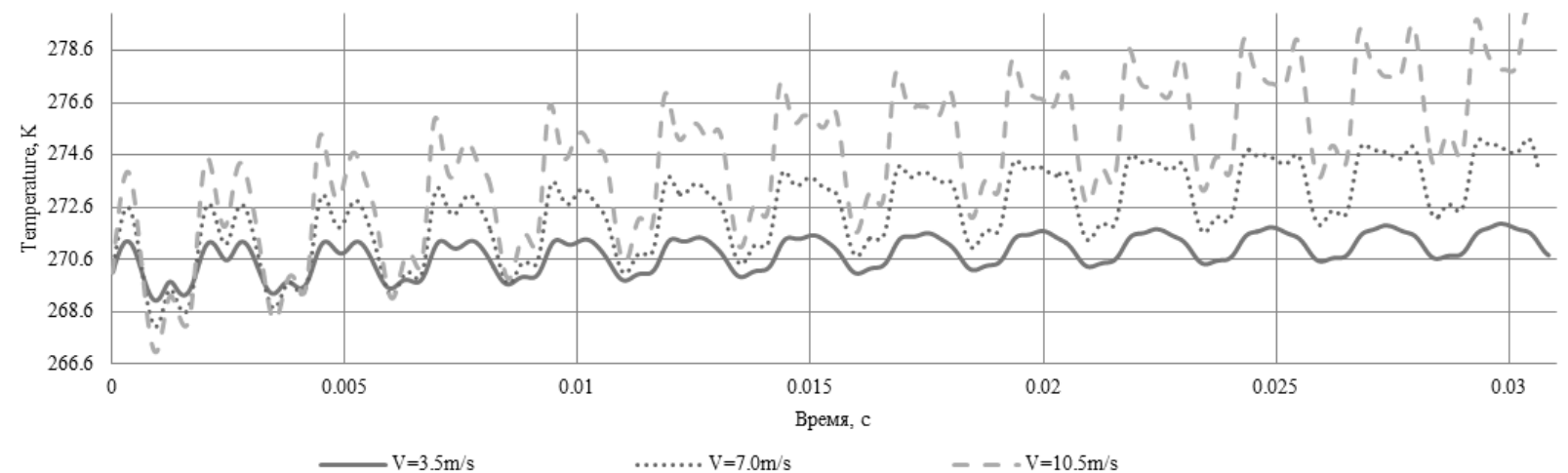

Fig.5. Temperature dependence of the LS gas-dynamic gap on time for various kinematic parameters.

Table 4. Temperature amplitude for LS different kinematic parameters.

\begin{tabular}{lccc} 
Table 4. Temperature amplitude for LS different kinematic parameters. & & \\
\hline Computational experiment & 1 & 2 & 3 \\
\hline $\begin{array}{l}\text { Propagation speed of gas } \\
\text { oscillations in the LS } \\
\text { circumferential direction, } \mathrm{m} / \mathrm{s}\end{array}$ & 3.5 & 7.0 & 10.5 \\
\hline Temperature amplitude $\mathrm{U}_{\mathrm{T}} \mathrm{K}$ & 271.9472 & 275.1519 & 279.8211 \\
\hline
\end{tabular}

\subsection{Analysis of the influence of the working fluid characteristics}

When studying the dependence of pressure oscillations in the LS gas-dynamic cavity on the working fluid characteristics the adiabatic index varied and was $1.1,1.25$ or 1.4 at density $\rho=1,29 \mathrm{~kg} / \mathrm{m}^{3}$.

With an increase in the adiabatic index from 1.1 to 1.4 , the pressure oscillations of the gas-dynamic cavity are observed, which have a periodic character (Fig. 6). The pressure amplitude is $102880.5 \mathrm{~Pa}$ for $\mathrm{k}=1.1$. For $\mathrm{k}=1.25$, the pressure amplitude is $102816.3 \mathrm{~Pa}$, and for $\mathrm{k}=1.4$ the pressure amplitude is $1003003 \mathrm{~Pa}$.

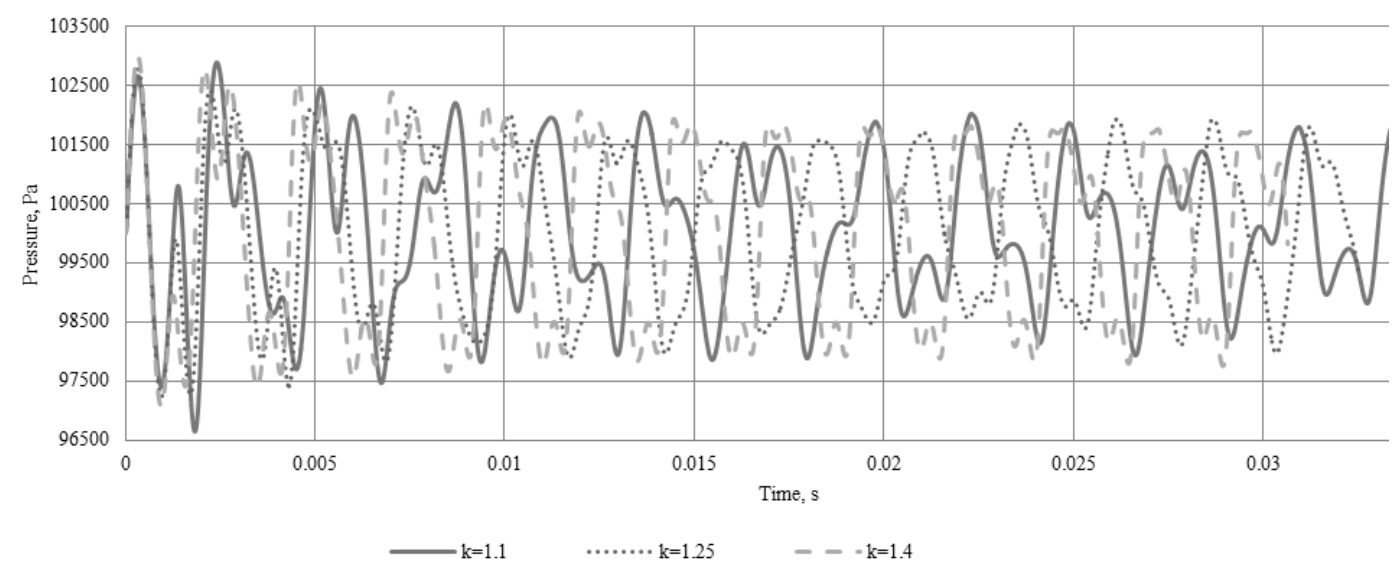

Fig.6. Pressure dependence of the LS gas-dynamic gap on time for different working fluid characteristics.

Table 5. Pressure amplitude for LS different working fluid characteristics.

\begin{tabular}{cccc}
\hline Computational experiment & 1 & 2 & 3 \\
\hline Adiabatic index, $\mathrm{k}$ & 1.1 & 1.25 & 1.4 \\
\hline $\begin{array}{c}\text { Maximum pressure amplitude } \\
\mathrm{U}_{\mathrm{P}, \mathrm{MPa}}\end{array}$ & 0.103 & 0.103 & 0.1 \\
\hline
\end{tabular}

When studying the dependence of temperature oscillations in the LS gas-dynamic cavity on the working fluidcharacteristics the adiabatic index varied and was $1.1,1.25$ or 1.4 , with density $\rho=1,29 \mathrm{~kg} / \mathrm{m}^{3}$.

With an increase in the adiabatic indexfrom 1.1 to 1.4, the temperature oscillations of the gas-dynamic cavity are observed, which have a periodic character (Fig. 7). The temperature amplitude is $271.8515 \mathrm{~K}$ for $\mathrm{k}=1.1$. For $\mathrm{k}=1.25$ the temperature amplitude is $274.0991 \mathrm{~K}$, and for $\mathrm{k}=1.4$ the temperature amplitude is $275.1212 \mathrm{~K}$. 


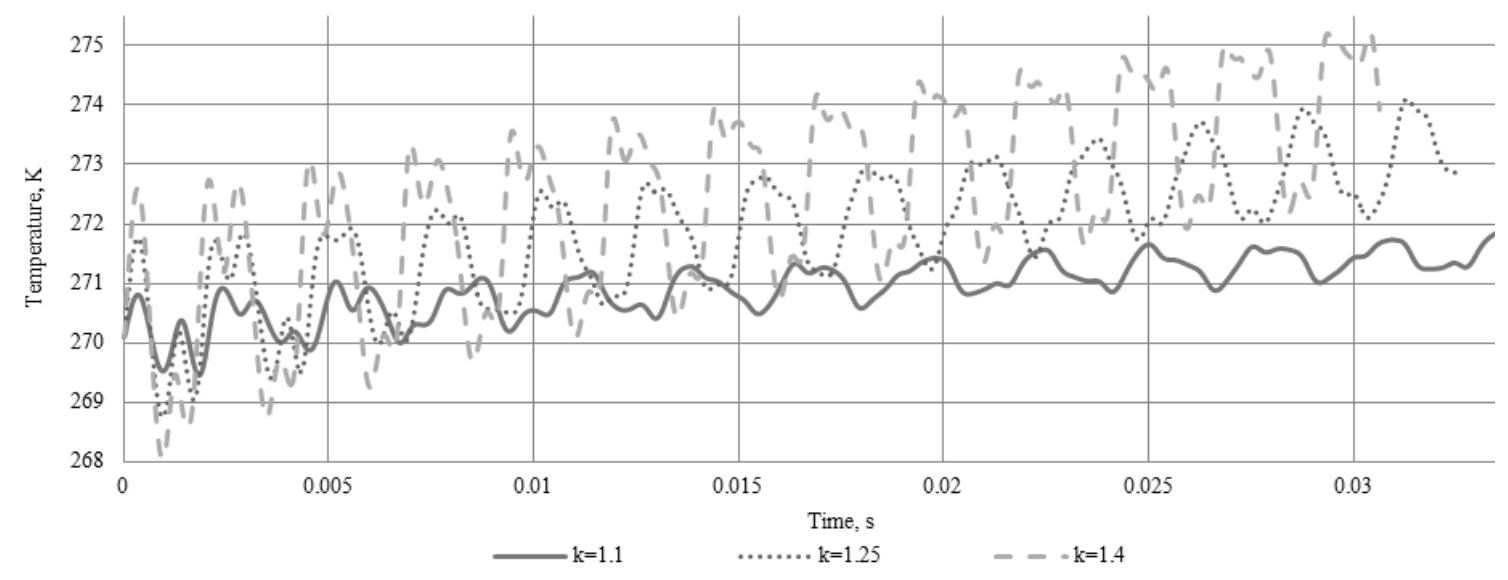

Fig.7. Temperature dependence of the LS gas-dynamic gap on time for different working fluid characteristics.

Table 6. Temperature amplitude for LS different kinematic parameters.

\begin{tabular}{cccc}
\hline Computational experiment & 1 & 2 & 3 \\
\hline Adiabatic index, $\mathrm{k}$ & 1.1 & 1.25 & 1.4 \\
\hline Temperature amplitude $\mathrm{U}_{\mathrm{T}}, \mathrm{K}$ & 271.8515 & 274.0991 & 275.1212 \\
\hline
\end{tabular}

\subsection{Analysis of the influence of physical and mechanical characteristics}

In the bi-directional aeroelastic formulation the LS calculation scheme was generated (Fig. 8) and a solution was obtained using equations (1-9) and initial and boundary conditions, which allowed obtaining the following results.

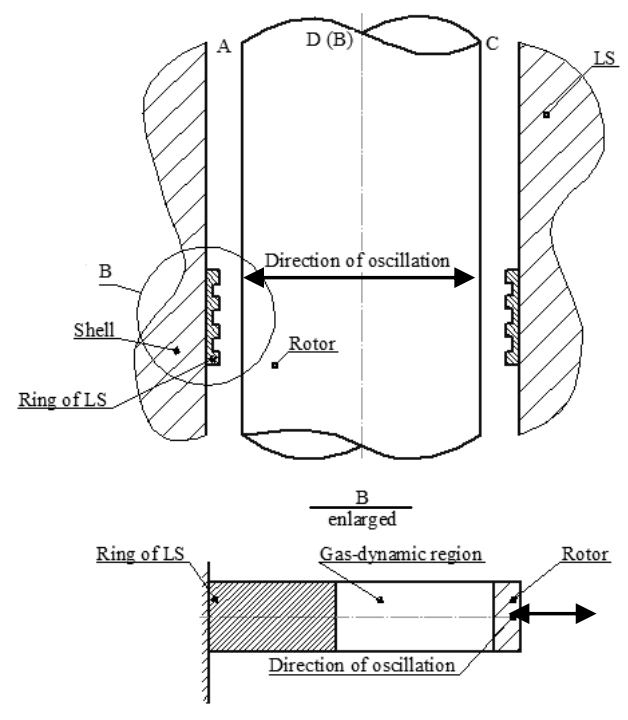

Fig. 8. Formation of the LS calculation scheme (bidirectional FSI-statement)

When studying the dependence of pressure oscillations in the LS gas-dynamic cavity on physico-mechanical characteristics of the LS the material density was set $\rho=7800 \mathrm{~kg} / \mathrm{m}^{3}$,Poisson ratio $\mu=0.35$, the elasticity modulus ranged within $50,100,150$, $200 \mathrm{GPa}$. With an increase in elasticity modulus from $50 \mathrm{GPa}$ to $200 \mathrm{GPa}$, the amplitude of the periodic pressure oscillations of the gas dynamic cavity decreases by 5 times. The pressure amplitude is $1 \mathrm{MPa}$ at E $=50 \mathrm{GPa}$. At E $=100 \mathrm{GPa}$ the pressure amplitude is $0.5 \mathrm{MPa}$, and at $\mathrm{E}=150 \mathrm{GPa}$ the pressure amplitude is $0.3 \mathrm{MPa}$, at $\mathrm{E}=200 \mathrm{GPa}$ the pressure amplitude is $0.2 \mathrm{MPa}$ (Fig. 9). Fig. 9 shows the dependence of pressure oscillations in the LS gas-dynamic gap on time for LS various physicomechanical characteristics.

When studying the dependence of displacements in the LS structure on physico-mechanical characteristics the density of the LS material was set $\rho=7800 \mathrm{~kg} / \mathrm{m}^{3}$, poisson ratio $\mu=0.35$, adiabatic index $\mathrm{k}=1.4$, air density $\rho=1.29 \mathrm{~kg} / \mathrm{m}^{3}, \mathrm{P}_{0}=0.1 \mathrm{MPa}$, the calculated ratio of cells in the structure to the total number of cells calculated $\mathrm{FL}=0.96$, the elastic modulus varied within 50 , 100,150 and $200 \mathrm{GPa}$. Near the gas-dynamic gap with an increase in E from $50 \mathrm{GPa}$ to $200 \mathrm{GPa}$ oscillations of displacements in the LS structure are observed. The oscillations are periodic in nature and stable in time. The displacement amplitude is $1 \times 10^{-2}$ microns at $\mathrm{E}=50 \mathrm{GPa}$. When $\mathrm{E}=100 \mathrm{GPa}$ the displacement amplitude is $5 \times 10^{-3} \mathrm{~m}$, and when $\mathrm{E}=150 \mathrm{GPa}$ the displacement amplitude is $3 \times 10^{-3} \mathrm{~m}$, for $\mathrm{E}=200 \mathrm{GPa}$ the displacement amplitude is $2.3 \times 10^{-3} \mathrm{~m}$ (Fig.10). Figure 11 shows the relationship of displacements in the LS structure versus time for LS different physico-mechanical characteristics. 


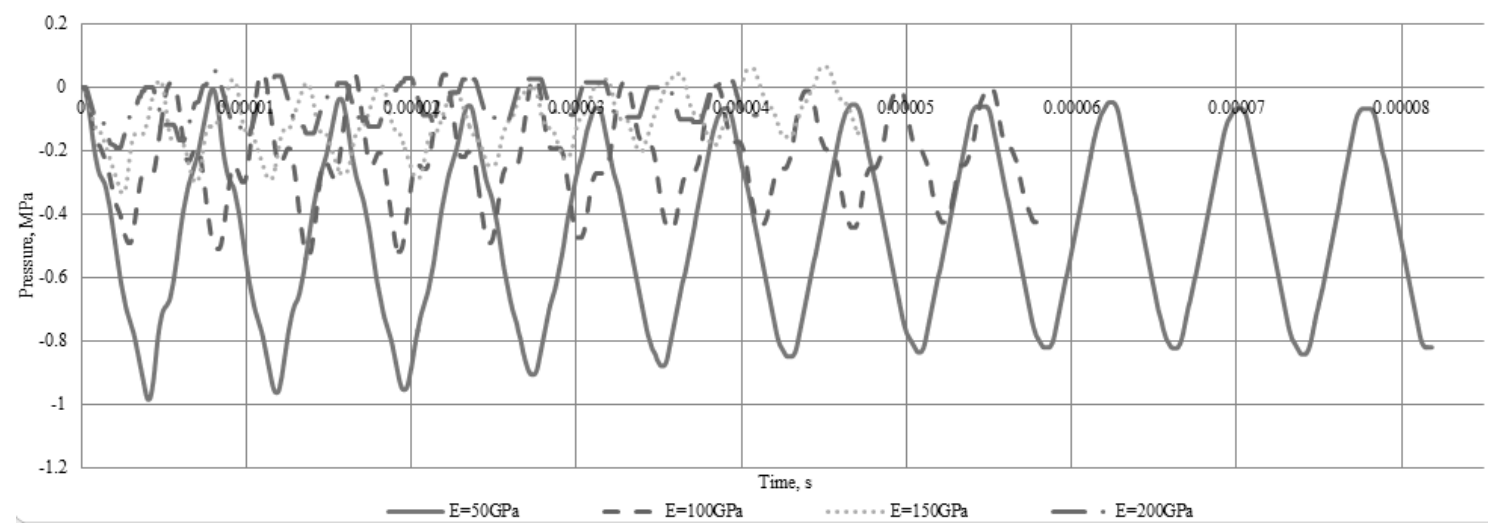

Fig. 9. Dependence of pressure oscillations in the LS gas-dynamic gap on time for LS various physico-mechanical characteristics.

Table 7. Amplitudeof pressure oscillations at different values of LS physico-mechanical characteristics.

\begin{tabular}{ccccc} 
Computational experiment & 1 & 2 & 3 & 4 \\
\hline Elasticity modulus E, GPa & 50 & 100 & 150 & 200 \\
\hline Pressure amplitude $\mathrm{U}_{\mathrm{P}, \mathrm{MPa}}$ & 1 & 0.5 & 0.3 & 0.2 \\
\hline
\end{tabular}

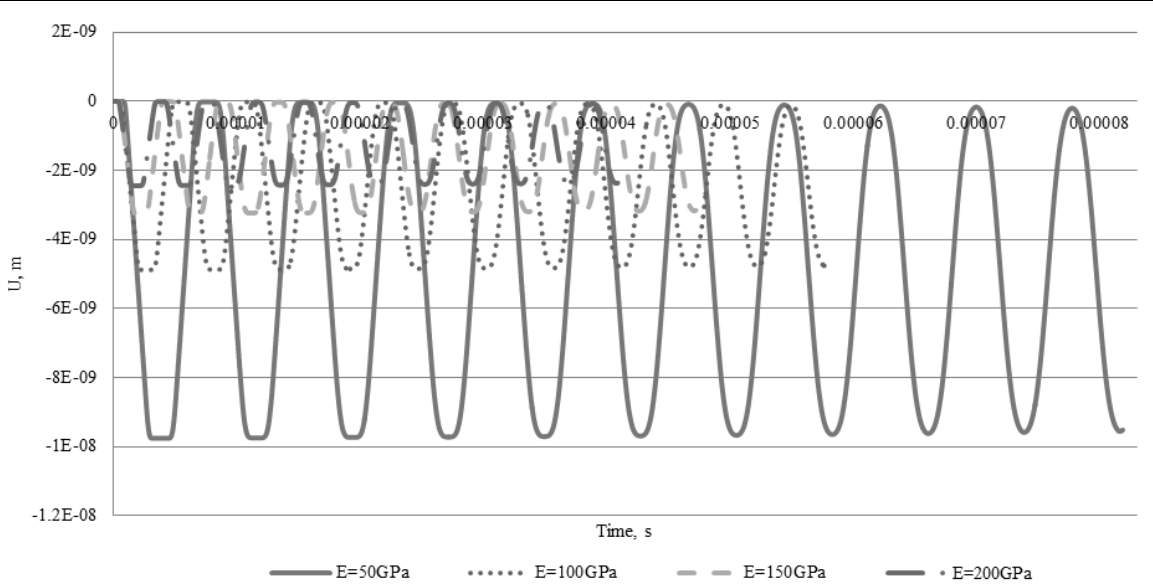

Fig. 10. Dependence of displacements in the LS structure versus time for LS various physico-mechanical characteristics.

Table 8. Displacements in the LS structure for LS different physico-mechanical characteristics.

\begin{tabular}{cccc}
\hline Computational experiment & 1 & 2 & 3 \\
\hline Elasticity modulus E, GPa & 50 & 100 & 150 \\
\hline Displacement amplitudeU $_{\mathrm{A}} \mathrm{m}$ & $1 \times 10^{-2}$ & $5 \times 10^{-3}$ & 200 \\
\hline
\end{tabular}

\section{Conclusions}

1. With an increase in the compression wave velocity arising from the approach of the rotor to the surface of the LS under vibrations from $3.5 \mathrm{~m} / \mathrm{s}$ to $10.5 \mathrm{~m} / \mathrm{s}$ the amplitude of the gas dynamic force increases from $23.6 \mathrm{H}$ to $45.5 \mathrm{H}$. The frequency does not change and is equal to $400 \mathrm{~Hz}$. At a natural rotor frequency of $808 \mathrm{~Hz}$, one can expect that with a minimum value of the gas flow velocity in the circumferential direction, weak vibrations may appear. As the speed increases, one can expect an increase in the LS vibrations.

2. With the shaft diameter increase from $65 \mathrm{~mm}$ to $260 \mathrm{~mm}$, the maximum amplitude of the gas dynamic force of $82.7 \mathrm{~N}$ is observed at a diameter equal to $130 \mathrm{~mm}$, the minimum amplitude of the gas dynamic force is observed at a diameter of $65 \mathrm{~mm}$ from $7.7 \mathrm{H}$, the frequency is $400 \mathrm{~Hz}$. The maximum frequency of the gas dynamic force is $770 \mathrm{~Hz}$ with a diameter of $65 \mathrm{~mm}$. The minimum frequency of the gas-dynamic force is $406 \mathrm{~Hz}$ with the diameter equal to $260 \mathrm{~mm}$. It can be seen that as the rotor diameter increases, the nominal values of the gas dynamic force increase. This is due to the increase in the rotor area at a constant nominal pressure. In this case the maximum amplitudes of gas-dynamic forces are observed when the oscillation frequency $f_{P}$ of the rotor is equal to the first natural frequency of the gas-dynamic pressure fluctuations of the circumferential cavity in the gap. Oscillation amplitude of gas-dynamic forces arelower at the rotor oscillation frequency $f_{P}$ equal to the second natural frequency of the gas-dynamic pressure oscillations of the circumferential cavity in the gap.Even lower are the amplitudes of gas-dynamic force oscillations at the rotor oscillation frequency $f_{P}$ equal to the fourth natural frequency of the circumferential pressure oscillations of the gas-dynamic cavity in the gap.The oscillation amplitudeof the gas-dynamic forces was also low at the natural frequency of the gas-dynamic cavity nonmultiple for the rotor frequency.

3. With an increase in the adiabatic index $\mathrm{k}$ from 1.1 to 1.4 the gas-dynamic force oscillation amplitude increases from $29.63 \mathrm{~N}$ to $35.15 \mathrm{~N}$, and the oscillation frequency of gas-dynamic forces decreases from $392 \mathrm{~Hz}$ to $388 \mathrm{~Hz}$. Thus, we note a weak influence of the working fluid characteristics on the LS vibrations. 
High-Performance Computing / L.N. Butymova, V.Ya. Modorskii

4. With increase in elastic modulus from 50GPa to 200GPa the pressure oscillation amplitude decreases from $0.97 \mathrm{MPa}$ to $0.19 \mathrm{MPa}$, pressure oscillation frequency rises from $134 \mathrm{kHz}$ to $256 \mathrm{kHz}$. Analysis of the influence of physical and mechanical characteristics on the LS vibrations demonstrated that with a hard material the strain rate is lower than with a soft material. The displacements in a softer material under given loads are $1 \times 10^{-2}$ microns. In a harder material the displacement amplitude is much lower.

5. By changing the rotor diameter, it is possible to reduce vibration. Thus, it is possible to reduce the gaps in the LS and reduce leakage.

\section{Acknowledgements}

The study was performed with a grant from the Russian Science Foundation (project №14-19-00877).

\section{References}

[1] Butymova LN. Effect of vibration on gas dynamics in modeling labyrinth seals of a gas transmittal unit centrifugal compressor. Bulletin of Perm National Research Polytechnic University. Aerospace technology 2016; 47: 243-259.

[2] Butymova LN, Modorsky VY, Petrov VY. Numerical modeling of the dynamic interaction in system "gas-structure" with harmonic motion of the piston in the variable section pipe. AIP Conference Proceedings 2016; 1770: 030103-1-030103-5.

[3] Butymova LN, Modorskii VY. One-way FSI simulation of the phase and the geometric parameters of the model of compressor blades on the oscillating gasdynamic processes pipe. MATEC the Web Conf. 2016; 75: $4 \mathrm{p}$.

[4] Butymova LN, Modorsky VY, Petrov VY. Numerical modeling the kinematic parameterseffect on the vibrations of the model compressor vanes in the system "gas-structure". Scientific and Technical Gazette Volga region 2015; 5: 157-160.

[5] Butymova LN, Modorsky VY, Shmakov AF. Experimental estimation of amplitude and phase characteristics of the interaction of gas-dynamic flow and structure. Scientific and Technical Gazette Volga Region 2014; 5: 127-129.

[6] Butymova LN, Modorsky VY. Investigation of gas-dynamic flow and structure in a model experimental setup. Bulletin of South Ural State University. Series: Computational Mathematics and Computer Science- 2014; 3(2): 92-100.

[7] Butymova LN, Modorsky VY. Study of oscillatory processes on resonant modes in the model apparatus. Scientific and Technical Gazette Volga region 2013; 6: 193-196.

[8] Butymova LN, Modorsky VY, Sokolkin YV. Development of experimental apparatus and investigation of the body material influence on the resonance frequencies in the "gas-structure". Scientific and Technical Gazette Volga Region 2013; 6: 197-200.

[9] Mekhonoshina EV, Modorsky VY. Development of numerical simulation techniques of the compressor aeroelastic operation. Scientific and Technical Gazette Volga Region 2014; 5: 264-268.

[10] Mekhonoshina EV, Modorsky VY. Impact of magnetic suspension stiffness on aeroelastic compressor rotor vibrations of gas pumping units. AIP Conference Proceedings 2016; 1770: 030113-1-030113-5.

[11] Makarov AA, Zaytsev NN. Engineering and theoretical problems of labyrinth seal applications in high-speed rotary machines. Herald PNRPU. Aerospace engineering 2015; 42: 61-81.

[12] Brikin BV, Evdokimov IE. Numerical simulation of the experiment on the flow in the labyrinth seal. Proceedings of the MAI \# 61. URL: http://mai.ru/ science / trudy / published.php ( 02/02/2017).

[13] Arbuzov IA, Tashkinov AA, Schenyatsky DV, Kirievsky BE, Bulbovich RV, Modorsky VY, Pisarev PV. Analysis of the impact of the entry apparatus in the connecting channel on the vibrational processes in the first stage of two-stage model pump. Scientific and Technical Gazette Volga $2012 ; 6$ : 108-111.

[14] Gaynutdinova DF, Modorsky VY, Shevelev NA. Experimental modeling of cavitation occurring at vibration. AIP Conference Proceedings 2016; 1770: 030111-1-030111-4.

[15] Shmakov AF, Modorsky VY. Numerical simulation of gas-dynamic, thermal processes and evaluation of the stress-strain state in the modeling compressor of the gas-distributing unit. AIP Conference Proceedings 2016; 1770: 030108-1-030108-5.

[16] Babushkina AV, Modorsky VY, Sipatov AM, Kolodyazhny DY, Nagorny VS. Modeling technique for the process of liquid film disintegration. AIP Conference Proceedings 2016; 1770: 030109-1-030109-7.

[17] Kalyulin SL, Modorsky VY, Paduchev AP. Numerical design of the rectifying lattices in a small-sized wind tunnel. AIP Conference Proceedings 2016; 1770: 030110-1-030110-4.

[18] Modorsky VY, Shevelev NA. Research of aerohydrodynamic and aeroelastic processes on PNRPU HPC system. AIP Conference Proceedings 2016; 1770: 030110-1-030110-4.

[19] Modorsky VY et al. Numerical study of actual problems of mechanical engineering and mechanics of solid and bulk materials by large particles method. A study of actual problems of mechanics and engineering. Moscow: National Academy of Applied Sciences, International Association of developers and users of the method of large particles 1995; 5: $1658 \mathrm{p}$.

[20] Modorsky VY, Shmakov AF, Butymova LN, Gainutdinova DF, Mekhonoshina EV, Kalyulin SL. Parallel calculation of dynamic processes in large-sized supercharger. Scientific service on the Internet: A variety of supercomputing worlds Proceedings of the International Supercomputer Conference. Russian Academy of Sciences Supercomputing Consortium of Russian Universities 2014: 258-262.

[21] Shmakov AF, Modorsky VY. Energy Conservation in Cooling Systems at Metallurgical Plants. Metallurgist 2016; 59(9): 882-886.

[22] Mekhonoshina EV, Modorskii VY. On a phase-shift of waves at the medium interface. Computer Optics 2015; 39(3): 385-391. DOI: 10.18287/0134-24522015-39-3-385-391. 